\title{
Influence of Laser Irradiation Times on Properties of Porous Silicon
}

Sabah M. Ali*
Alwan M. Alwan*

Oday A. Abass*

Date of acceptance 8/10/2007

\begin{abstract}
:
Porous silicon (P-Si) has been produced in this work by photoelectrochemical (PEC) etching process. The irradiation has been achieved using diode laser of $(2 \mathrm{~W})$ power and $810 \mathrm{~nm}$ wavelength. The influence of various irradiation times on the properties of P-Si material such as $\mathrm{P}-\mathrm{Si}$ layer thickness, surface aspect, pore diameter and the thickness of walls between pores as well as porosity and etching rate was investigated by depending on the scanning electron micrograph (SEM) technique and gravimetric measurements.
\end{abstract}

Keywords: Porous Silicon, Laser beam, SEM Images.

\section{Introduction:}

Porous silicon $(\mathrm{P}-\mathrm{Si})$ can be considered as a silicon crystal having a network of voids in it. The nanosized voids in the silicon bulk result in a sponge-like structure of pores and channels surrounded with a skeleton of crystalline silicon nanowires. The structural properties of porous silicon are fundamentally determined by the diameter of pores and thickness of P-Si layer. These parameters depend on preparation conditions $[1,2,3]$.

Crystalline silicon (C-Si) is well studied for production of electronic components, but it is useless for fabrication of light-emitting devices, because it possesses indirect nature of the fundamental bandgap $[2,4]$.

Canham [1] has reported in 1990, observation of bright photoluminescence (PL) from P-Si material even at room temperature. Therefore, PSi material has become a popular material among scientists and technologists, and has been applied in various fields during the past two decades.
Laser-assisted wet etching (LAWE) has been investigated for microelectronics fabrication. LAWE offers some possible advantages, such as, processing at relatively low temperatures, mask less pattering and beam-controlled anisotropy [5]. The structural characteristics of porous silicon have been studied by a range of techniques. Direct images of the material can be carried out by scanning electron microscopy [6].

Lehmann and Gosele [7] have reported that the diameter of pores or channels of the PSi layer is ranging from 1 up to $100 \mathrm{~nm}$ and the porosity of $(20-80 \%)$.

In this work, we have studied the influence of different irradiation times of laser radiation on the structural properties of n-type P-Si layer during the etching process.

\section{Experiment:}

Commercially available mirrorlike n-type (111) oriented silicon wafers of $(500 \pm 15 \mu \mathrm{m})$ thickness with resistivity $(\rho=0.02 \Omega . \mathrm{cm}$ ) respectively have been used as substrate. Before

*School of Applied Sciences/University of Technology/Department of Applied Physics/

Branch of Laser/Baghdad-IRAQ.Email: *oday_laser@yahoo.com 
Photoelectrochemical (PEC) etching process, the silicon wafer has been cut out into small pieces in dimensions of (1 $\times 1 \mathrm{~cm})$. These pieces were rinsed with ethanol to remove dirt followed by etching in dilute (10\% wt.) hydrofluoric (HF) acid to remove the native oxide layer. The photoelectrochemically etched area for all samples has been 0.6 $\mathrm{cm}^{2}$.

We deposited aluminum film on the front side of silicon sample in order to creating an ohmic contact. This has been achieved by using thermal evaporation process in vacuum chamber $\left(2 \times 10^{-5}\right.$ torr $)$.

The simple set-up of PEC etching process consisted of one commercially available $\mathrm{CW}$ diode laser with power density $(2.33 \mathrm{~W})$ and $(810 \mathrm{~nm})$ wavelength, a dual Farnel LT 30/2 power supply as a current source, digital multimeter and an ethanoic solution of $25 \% \mathrm{HF}$ is obtained by 1 volume of ethanol and 1 volume of $48 \%$ wt. Hydrofluoric (HF) acid. The HF concentration in the ethanoic solution is given by $((1 \times 49 \%) /(1+1)=25 \%)[6]$. Figure (1) depicts a schematic diagram of the PEC set-up. The sample has been mounted on a Teflon cell in such a way that current should not pass from the back surface. At the same time the silicon was mounted as an anode and the electrical circuit was completed by putting a platinum electrode as a cathode in a parallel way to achieve the homogeneous layers of n-type silicon. The value of the utilized current density for PEC etching process has been (30 $\mathrm{mA} / \mathrm{cm}^{2}$ ).

Simultaneously, the samples have been irradiated with laser radiation. The distance between the laser source and the sample was (25 $\mathrm{cm})$. The irradiation times varies from 1 to 15 minutes.
The structural properties of P-Si layer such as surface aspect, pore diameter, thickness of walls between pores and layer thickness have been studied in this work by using a scanning electron micrograph (SEM) (Leo-1550). The given voltage for each photo was (5KV). The SEM measurements were carried out in the (Institute for Bio-and Nano-systems (IBN2)-Germany). The porosity of $\mathrm{P}-\mathrm{Si}$ layer has been calculated by measuring the weight of the samples before and after etching process as well as the weight of sample after removing of PSi layer from sample and using the following equation $[4,8]$ :

$\gamma=\frac{M_{1}-M_{2}}{M_{1}-M_{3}}$.

where $M_{1}(\mathrm{gm})$ and $M_{2}(\mathrm{gm})$ are the weights of silicon sample before and after etching process respectively. $M_{3}$ (gm) is the weight of the silicon sample after removing of PSi layer by immersing the sample in $\mathrm{KOH}$ solution for 10 minutes.

The etching rate $(v)$ can be measured using the following equation [6].

$v=\frac{d}{t}$.

where $v(\mu \mathrm{m} / \mathrm{min})$ is the etching rate, $d$ $(\mu \mathrm{m})$ is the layer thickness and $t(\mathrm{~min})$ is the etching time.

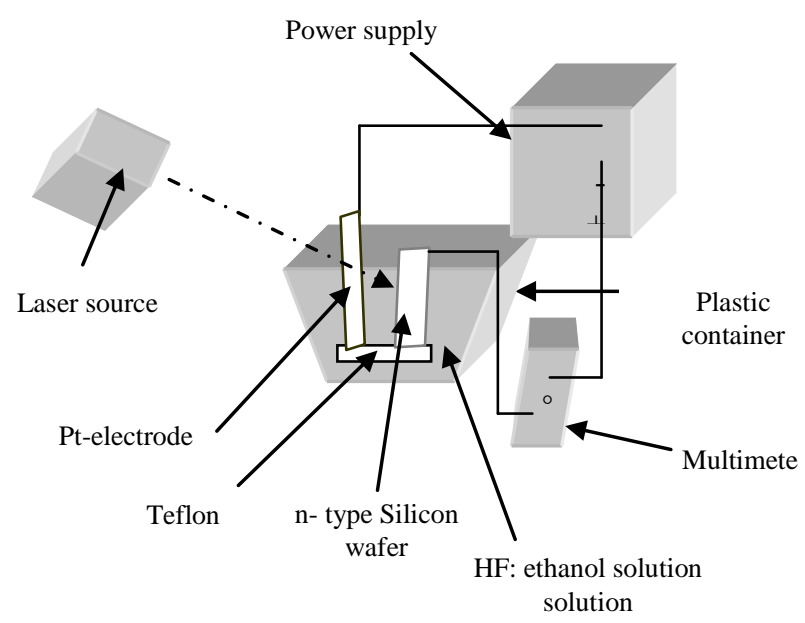

Fig. (1); Schematic diagram depicts the PEC etching process. 


\section{Results and discussion:}

For clarifying these sketched data in figures $(3,4)$ which plotted according to SEM images, we believes that the simple one-dimensional model suggested by Choy and Cheah [8] acceptable for our cases. We are choosing only three SEM images for three samples, so as clear the role of etching time on porous silicon formation.

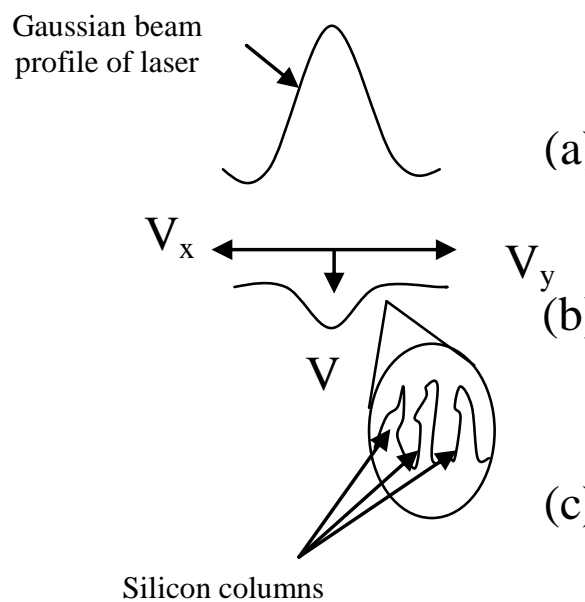

Fig. (2); A schematic diagram depicts the etching process [8].

Figure (2-a,b,c), depicts schematically the etching process; figure (2a) represents the Gaussian profile of a laser beam, while figure (2b) shows the profile of an etched spot on the silicon sample, and figure (2c) represents the microscopic structure inside the etched area. At the beginning of the etching process, the wafer is etched in three directions; two spreading out along the surface from the center of an etched site, and one into the silicon wafer, and the corresponding rates are designated as $V_{x}, V_{y}$ and $V_{z}$, respectively. The etching rate $V_{x}$ is assumed to be the same as $V_{y}$ and less vigorous than that of $V_{z}[8,9]$.

This behavior can be attributed to the distribution of the laser beam
(Gaussian mode). Therefore the etching rate at the center of irradiated area is higher than the others because it received the most intense light. As a consequence the numbers of required holes for initiating the reaction between irradiated area and HF acid in the center would be more than that in other positions [9].

If a hole $\left(\mathrm{h}^{+}\right)$reaches the surface, nucleophilic attack on $\mathrm{Si}-\mathrm{H}$ bonds by fluoride ions $\left(\mathrm{F}^{-}\right)$can occur and a $\mathrm{Si}-\mathrm{F}$ bond is established. Due to the polarizing influence of the bonded $\mathrm{F}$, another $\mathrm{F}^{-}$ion can attack and bond under generation of an $\mathrm{H}_{2}$ molecule and injection of one electron into the bulk silicon. Because of the polarization induced by the $\mathrm{Si}-\mathrm{F}$ groups, the electron density of the $\mathrm{Si}-\mathrm{Si}$ back bonds is lowered and these weakened-bonds will now be attacked by $\mathrm{HF}$ or $\mathrm{H}_{2} \mathrm{O}$ in a way that the silicon surface atoms remain bonded to hydrogen When a silicon atom becomes removed from an atomically flat surface by this reaction, an atomic size dip remains. This change in surface geometry will change the inert electric field distribution in such a way that holes transfer occurs at this location preferentially. Therefore pores of about $1 \mu \mathrm{m}$ will establish in a few minutes on polished n-type silicon surface by this process $[6,7,8]$.

Figures (3) and (4) show the relation between layer thickness and etching rate with etching time, for the prepared P-Si layer at various etching times (1-15 min) with fixed current density of $30 \mathrm{~mA} / \mathrm{cm}^{2}$ on $(0.02 \Omega . \mathrm{cm}) \mathrm{n}$ type silicon substrates resistivity, immersed in $25 \%$ HF concentration. 


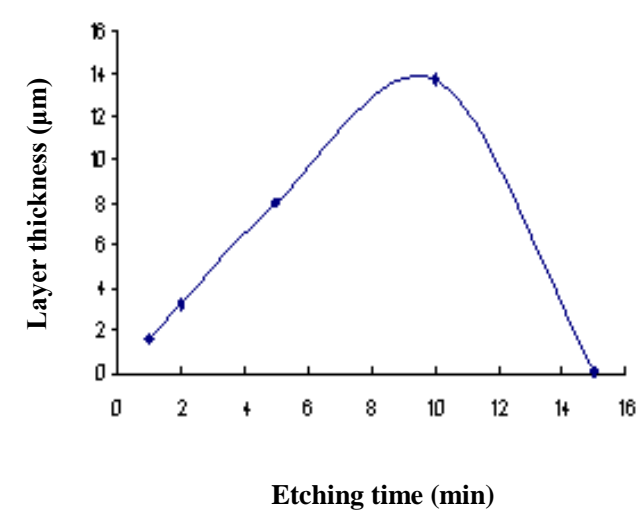

Fig. (3); The relation between Layer thickness and Etching time.

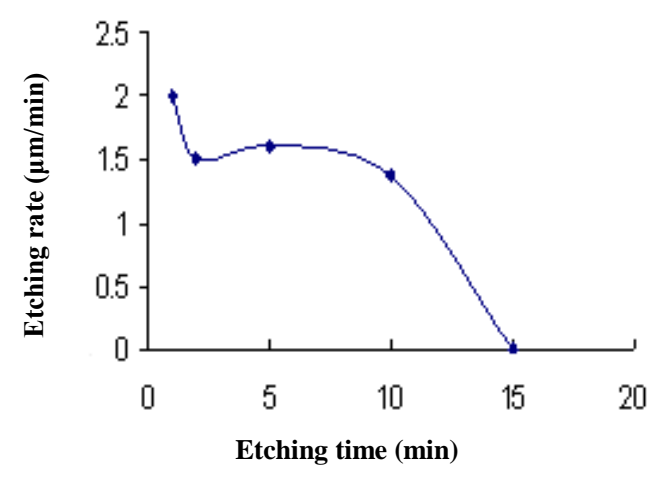

Fig. (4); The relation between the etching rate and etching time.

From these figures one can observe that the PSi layer is formed with thickness $\mathrm{D}$ after short time $(\mathrm{t}=1$ min) as shown in figure (3). Layer thickness has been thinner than the absorption depth of the incident laser. Therefore, etching rate $\left(V_{z}\right)$ will remain constant and vigorous at this time as shown in figure (4). While, the lateral etching rates $\mathrm{V}_{\mathrm{x}}$ and $\mathrm{V}_{\mathrm{y}}$ will slow down in comparison with etching rate $\left(V_{z}\right)$ as shown in figure (2).

For a longer etching times $(t=5)$, a PSi layer thickness D increases and the concentration of the charge carriers changes as shown in figures (3) and (5a) and also the etching rate $\left(V_{z}\right)$ decreases as shown in figure (4). The thickness of P-Si layer becomes too long at irradiation time $(\mathrm{t}=10 \mathrm{~min})$ as shown in figures (3) and (6-a), for the incident light to reach the base of the silicon columns.

Therefore with increasing of etching time to $(t=15 \mathrm{~min})$, the etching rate $\left(\mathrm{V}_{\mathrm{z}}\right)$ would be reduce gradually until is stopping as shown in figure (4). At this stage, the sideways etching process at the surface would become dominant and result in a gradual erosion of the silicon columns. When the columns become shorten sufficiently, light can reach the lower part of the silicon columns again and causes generation of electron-hole pairs and then the etching of the silicon substrate restarts again, and columns are formed as shown in figure (7-a).

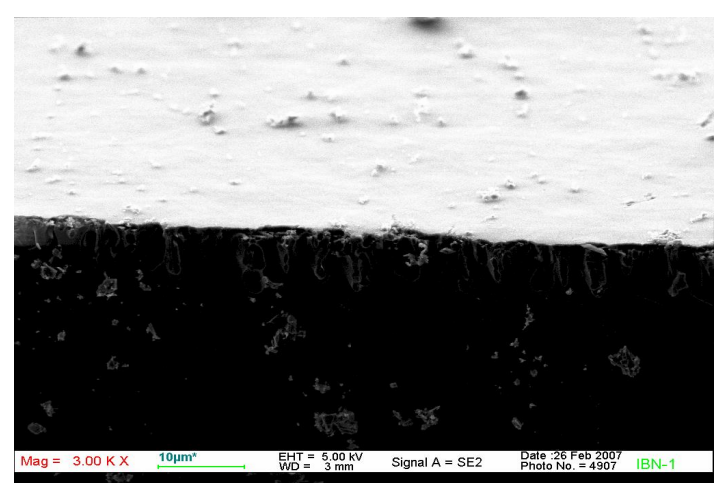

(a)

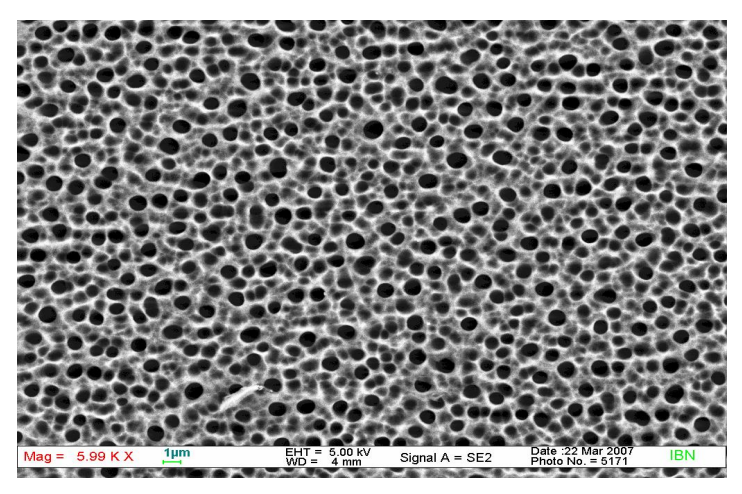

(b)

Fig. (5); SEM Images of P-Si layer prepared at 5 min etching time, aedge-view and b- top-view. 


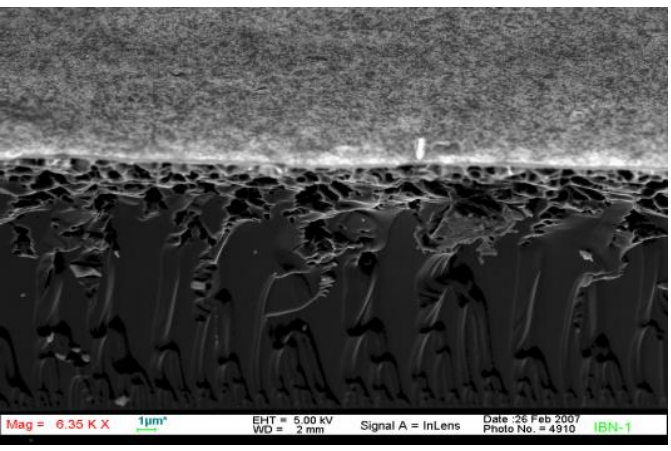

(a)

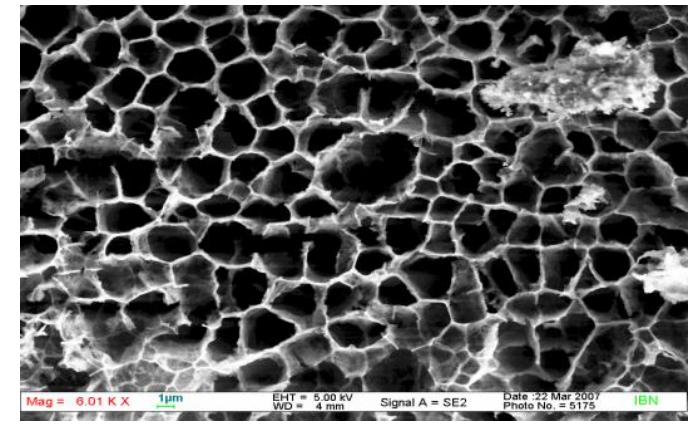

(b)

Fig. (6); SEM Images of P-Si layer prepared at $10 \mathrm{~min}$ etching time, aedge-view and b- top-view.

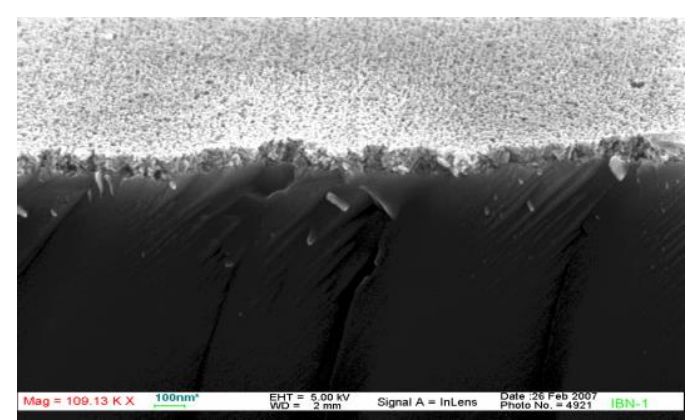

(a)

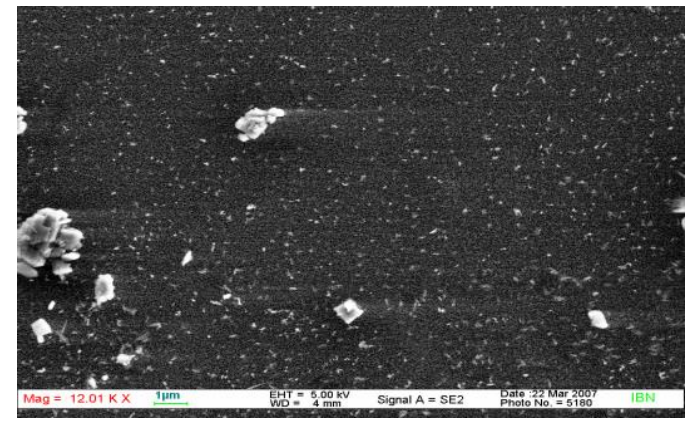

(b)

Fig. (7); SEM Images of P-Si layer prepared at $10 \mathrm{~min}$ etching time, aedge-view and b- top-view.

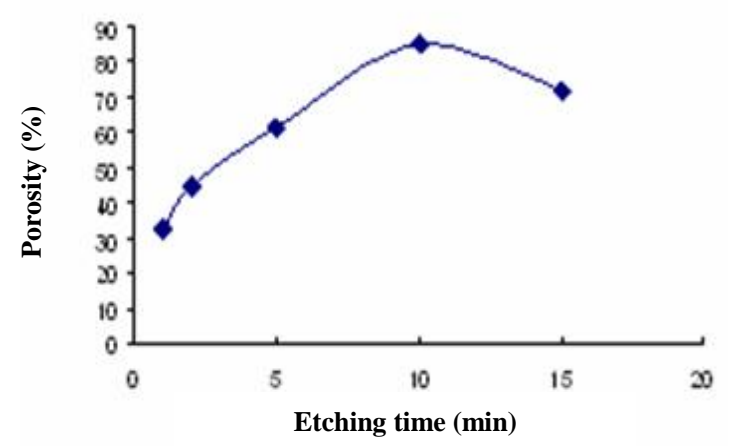

Fig. (8); Effect of etching time on porosity.

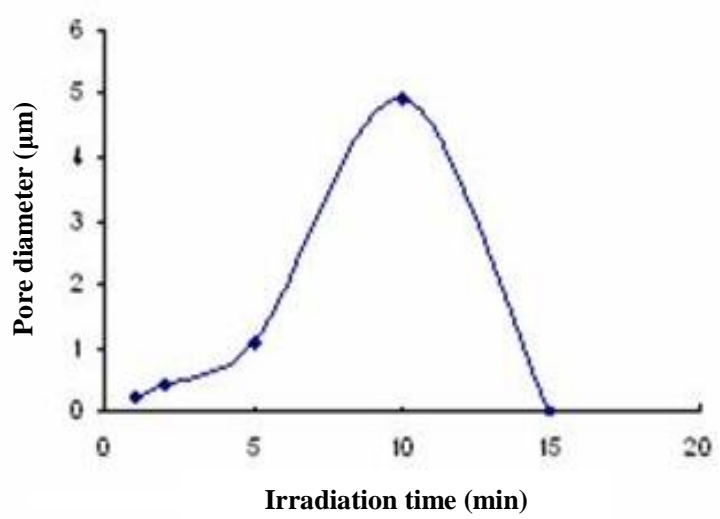

Fig. (9); Effect of etching time on pore diameter.

From previous SEM images and described results in figures (8 and 9), we can see the values of porosity and pore diameter are increasing with increasing of etching time. This largeness in pore width may be attributed to increasing of holes number on surface of silicon electrode with etching time which leads to preferential dissolution between nearest-neighbor pores, thereby promoting the pore-pore overlap $[7,10,11]$. As etching process proceeds, extra holes reach the surface leading to further dissolving of the walls between pores and then the nearest pores would be combined (pore dying) as shown in figure (8), leading to fraction amission of void from PSi layer continuously until losing all pores and then initiating 
new pores with narrow diameter, consequently the porosity becomes lower as shown in figure (9).

By depending on these results and according to definition of porosity, which define as the fraction of void within the P-Si layer $[4,12,13]$. We can confirm that the porosity depends on number and diameter of pores which itself depends on etching time.

\section{Conclusion:}

In light of these results and facts which have been mentioned previously in this work, the photoelectrochemical etching process could consider as a very useful technique in production $\mathrm{P}-\mathrm{Si}$ material.

The morphological properties of the formed P-Si material via PEC etching process have been dramatic and very sensitive to etching. In general, the porosity, pore width, and thickness of $\mathrm{P}$ Si layer have increased with increasing of etching time. On the other hand the etching rates, layer thickness and porosity have maximum values at an etching time 10 minute.

\section{ACKNOWLEDGEMENT}

We are grateful to Dr. Hans Bohn for helpful discussions. This work supported by institute for Biologic and Nano-systems (IBN2), Germany.

\section{Reference:}

1- Canham L. T., (1990). Silicon quantum wire array fabrication by electrochemical and chemical dissolution of wafers. Appl. Phys. Lett., 57: 1048 .

2- Andrea P., (2005), Investigation of pristine and oxidized porous silicon, Msc. Thesis, University of Oulu, Finland.
3- Lee W. H., Lee C., Jang J., (1996). Quantum size effects on the conductivity in porous silicon. Journal of Nona-Crystalline Solids., 200: 914.

4-Lehmann V., Foll H., (1990). Formation Mechanism and Properties of Electrochemically Etched Trenches in n-Type Silicon. J. Electrochem. Soc., 137: 659.

5- Thonissen M., Berger M. G., ArensFischer R., Gluck O., Kruger M., Luth H., (1996). Illumination-assisted formation of Porous Silicon. Thin solid films., 276: 24.

6- Canham L. T., (1998). Properties of porous silicon; INSPEC, England.

7- Lehmann V., Gosele U., (1991). Porous Silicon Formation: A quantum Wire Effect. Appl. Phys. Lett., 58: 858. 8- Choy C. H., Cheah K. W., (1995). Laser-induced etching of Silicon. Appl. Phys. A., 61: 50.

9- Noguchi N., Suemune I., (1993). Production of porous silicon by laserinduce etching. Appl. Phys. Lett., 26: 1433.

10- Lim P., Brock J. R., Trachtenbreg I., (1992). Laser-induced etching of Silicon in hydrofluoric acid. Appl. Phys. Lett., 60: 488.

11- Lee S., Kim D. Y., (2005). Effect of Incoherent Light Illumination during Electrochemical Anodization on the Formation and the Properties of Porous Si. Journal of the Korean Physical Society., 47: 889.

12- Vincent V. D., Reginald M. P., Michael J. S., (1993). Enhanced Photoemission from Short-Wavelength Photochemically Etched Porous Silicon. J. Phys. Chem., 97: 4508.

13- Matthias S., Muller F., Gosele U., (2005). Controlled nouniformity in macroporous silicon pore growth. Appl. Phys. $\quad$ Lett., 87: 4106. 
تأثير أزمنة تشعيع الليزر على خواص السليكون المسامي

عدي أركان عباس

علوان محد علوان

صباح محمد عليث

|الجامعة التكنلوجية ـ قسم العلوم النطبيقية ـ فرع الليزر

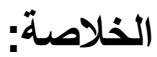

تم تحضيرالسليكون المسامي باستخدام عملية القشط الضوئي-الكهروكيميائي. التشعيع اجري باستخدام

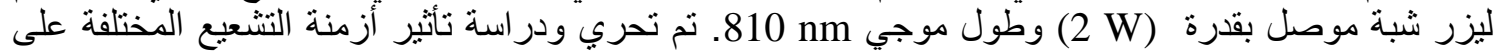

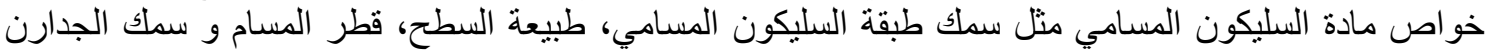

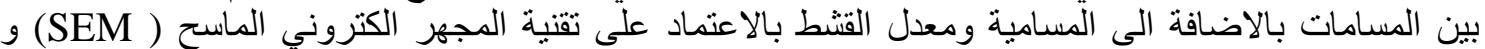

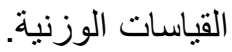

